Article

\title{
Between a Troll and a Hard Place: The Demand Framework's Answer to One of Gaming's Biggest Problems
}

\author{
Christine L. Cook \\ Department of Communication and Cognition, Tilburg University, 5037AB Tilburg, The Netherlands; E-Mail: c.l.cook@uvt.nl
}

Submitted: 7 August 2019 | Accepted: 27 October 2019 | Published: 20 December 2019

\begin{abstract}
The demand framework is commonly used by game scholars to develop new and innovative ways to improve the gaming experience. However, the present article aims to expand this framework and apply it to problematic gaming, also known as trolling. Although still a relatively new field, research into trolling has exploded within the past ten years. However, the vast majority of these studies are descriptive in nature. The present article marries theory and trolling research by closely examining interdisciplinary empirical evidence from a single platform-video games-and applying the various forms of demands to propose a testable, dual-route model of trolling behaviour. Within the video game context, I argue the presence of two primary causal mechanisms that can lead to trolling: 1) Demand imbalance between players and the game; and 2) demand imbalance between players. The article discusses how these two types of imbalance can lead to trolling, which kinds of demands can be imbalanced, and how future researchers can use the demand framework to expand our understanding of trolling.
\end{abstract}

\section{Keywords}

demand imbalance; flow theory; motivations; multiplayer online games; trolling

\section{Issue}

This article is part of the issue "Video Games as Demanding Technologies" edited by Nicholas David Bowman (Texas Tech University, USA).

(C) 2019 by the author; licensee Cogitatio (Lisbon, Portugal). This article is licensed under a Creative Commons Attribution 4.0 International License (CC BY).

\section{Introduction}

The demand framework presented in Bowman's (2018) overview has been a useful tool for games scholars in recent years, explaining gamers and their behaviours by relating them to four key demands: cognitive, emotional, social, and physical. However, the majority of studies examining the impact demands have on gamers' choices both in-game and out deal with optimization. Games' cognitive demands, for example, are studied for their potential benefits for cognitive training and interventions (Boot, Kramer, Simons, Fabiani, \& Gratton, 2008; Bowman \& Tamborini, 2012; Green, 2018). Although this research is beneficial for many gamers, in reality, not all gamers are striving for an optimal experience. In fact, there has been a recent explosion of literature concerning gamers who seem to seek the opposite for both themselves and other players in the game: trolls (Cook, Schaafsma, \& Antheunis, 2018; Thacker \& Griffiths, 2012).

Though trolling as a whole is still a widely misunderstood phenomenon rife with contradicting definitions and conflicting accounts of motivations and reactions (for complete discussions see Cook et al., 2018; Dynel, 2016), trolling specific to gaming platforms is a much clearer concept. Comprising behaviours traditionally associated with griefing culture (Chesney, Coyne, Logan, \& Madden, 2009; Coyne, Chesney, Logan, \& Madden, 2009) as well as more lighthearted jabs and jokes (Cook et al., 2018; Thacker \& Griffiths, 2012), trolling is an umbrella term that refers to the instrumental use of game, website, or chat mechanics at another person's expense, though it finds its roots in both practical jokes and boundary maintenance practices in niche communities (see Graham, 2019). The mechanics that trolls use are the very same mechanics that create a game's demands. 
The same vocal channel that a Counter Strike: Global Offensive player uses to strategize with his team-a social demand of the game-a troll uses to spam his teammates with an off-key rendition of Jingle Bells. While a skilled player might practice their aim for hours to land a kill in League of Legends, thus meeting the game's cognitive and physical demands, a troll might fling their avatar in front of a poorly-aimed shot in order to die, thus disadvantaging their team (for additional examples, see Table 1). Trolls can even operate outside of the game via online streaming websites like Twitch.tv to emotionally victimize a vulnerable streamer (Johnson, 2019) or even another audience member (Seering, Kraut, \& Dabbish, 2017). Although there are accounts of trolling being an enjoyable activity, usually between friends (for examples see Cook et al., 2018), it is generally considered an issue that has been plaguing online gaming since its inception. The present piece proposes demand imbalance as a major cause of this choice of playstyle, and aims to explain how the very same demands in the very same game can cause one player to flourish, and another to rebel.

\section{Conceptualizing Demands and Trolling in Games}

As previously stated, games produce four key demand types: cognitive, social, emotional, and physical (Bowman, 2018). Exact theoretical definitions for these demand types are presented in Table 1, as well as examples of how these demands could be a part of trolling practices. These demands are typically treated as continuous dimensions, as evidenced by the Video Game Demand Scale (Bowman, Wasserman, \& Banks, 2018).

According to Bowman et al. (2018), interactivity is the key to their operationalization. It is the constant, two-way interaction between the player and the game mechanics that creates demand, explaining at least in part how different demand levels can be produced in different players by the same game mechanics. However, it is much easier to operationalize a game mechanic than an interaction, and so most demand scholars refer more heavily to mechanics over demands (for examples see Eden, Ewoldsen, Lee, \& Beyea, 2018; Green, 2018;
Possler, Klimmt, \& Raney, 2018). Despite the mechanics being the simpler aspect to understand and describe, and thus more frequently referenced in demand literature, demands themselves are actually the bi-directional relationship between the player and the mechanics, as visualized in Figure 1.

The top half of Figure 1 illustrates the basic relationships that make up the demand framework, but we cannot explain trolling without implicating the result of these interactions: in-game behaviour. Behaviour here is conceptually defined as any action taken by a player within a game's user interface. This definition not only neatly encompasses all actions that could be taken in a game, such as killing an enemy or solving a puzzle, but also covers any action that could be defined as trolling within a gaming context, such as harassing another player via chat or purposely killing a teammate (see Cook et al., 2018). Physical demands present perhaps the most obvious case of behaviour resulting from in-game demands: Your avatar needs to get to the other side of the screen to save a captured princess, so you press the appropriate buttons on your controller to make that happen. However, there is a hidden premise underlying the previous statement: Your goals match the predetermined goals given by the game developer. This is an important assumption that goes largely unmentioned in most demand-based literature, but scaffolds most of the optimization findings presented in extant literature. You meet or exceed the demands the game presents only if you want to win the game; there is no need to train our multitasking capabilities (Green, 2018) or feel guilty about murdering innocents (see No Russian mission in Call of Duty: Modern Warfare 2) if we do not care about reaching the end of the game. Thus, in order to understand how demand theory can explain trolls and trolling, we first have to understand trolls' goals.

\subsection{Goal Alignment and Co-Creation in Relation to Trolling Practices}

According to existing trolling research, trolls' goals only sometimes align with that of developers (Buckels,

Table 1. Definitions of demands as presented by Bowman (2018).

\begin{tabular}{|c|c|c|}
\hline Demand & Definition & Trolling Example \\
\hline Cognitive & $\begin{array}{l}\text { "The extent to which the user is required to implicitly or ex- } \\
\text { plicitly rationalize or understand the game" (p. 5). }\end{array}$ & $\begin{array}{l}\text { Increasing cognitive demand by thwarting } \\
\text { one's team's strategy to win by purposely } \\
\text { killing one's own character. }\end{array}$ \\
\hline Emotional & $\begin{array}{l}\text { "The extent to which a video game causes the user to have } \\
\text { an implicit or explicit affective response to the game" (p. 8). }\end{array}$ & $\begin{array}{l}\text { Increasing emotional demand by hurling per- } \\
\text { sonal insults at a teammate or enemy. }\end{array}$ \\
\hline Social & $\begin{array}{l}\text { "The extent to which a system (or actor) triggers an implicit } \\
\text { or explicit response in the user to the presence of other so- } \\
\text { cial actors" (p. 13). }\end{array}$ & $\begin{array}{l}\text { Increasing social demand by spamming in the } \\
\text { chat window. }\end{array}$ \\
\hline Physical & $\begin{array}{l}\text { "The extent to which a system requires the user to exert } \\
\text { discrete or holistic physical effort" (p. 11). }\end{array}$ & $\begin{array}{l}\text { Reducing physical demand by abusing a glitch } \\
\text { to become invulnerable in-game. }\end{array}$ \\
\hline
\end{tabular}




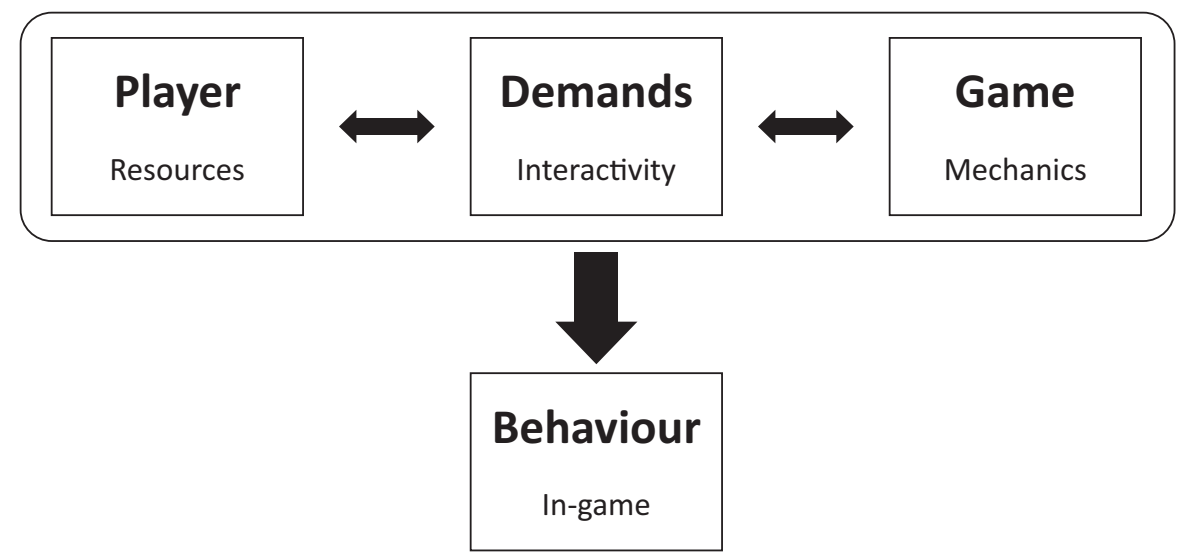

Figure 1. The author's interpretation of the demand framework as it is conceptualized in Bowman (2018). Note: Demands function as an interaction between a player's resources and the game's mechanics, producing the player's in-game behaviours.

Trapnell, \& Paulhus, 2014; Cook et al., 2018; Herring, Job-Sluder, Scheckler, \& Barab, 2002; Shachaf \& Hara, 2010; Thacker \& Griffiths, 2012). Trolling behaviours that are motivated by revenge or interaction seeking (Cook et al., 2018; Thacker \& Griffiths, 2012), for example, can simply ignore the demands presented by the game, using the mechanics to achieve goals independent to the game's (i.e., making a friend or giving a troll a taste of their own medicine). In this case, trolling is a breakdown of the co-creation that typifies normal game play (Bowman, 2018). Instead of the aligned goals implicit to traditional demand theory work-Situation $A$ in Figure 2-trolling motivated by personal goals that do not involve winning the game looks more like Situation C (Figure 2). In essence, goal alignment determines just how demanding a game's demands are to a player; with complete alignment, players work to meet the game's demands and co-create the gaming experience, while with no alignment, the co-creation process breaks down completely.

Even so, Situation B, in which the developer and player's goals align only partially, remains unexplained. How common is this situation? Trolling literature would suggest that, with deviant players, this is actually the most common option from those listed in Figure 2 (Cook et al., 2018). According to Cook et al. (2018), the number one motivation for trolling is personal enjoyment, and the most popular catalyst for trolling is feeling bored or 'tilted,' which is to say in a negative mood or headspace. Given that games are usually considered a leisure activity designed to alleviate boredom and promote enjoyment (Daneels, Vandebosch, \& Walrave, 2019), it would seem that the trolls' goal to have fun is at least partially aligned with developers' goal to create a game that fills that need. Of course, this is not the case for all games and all developers, but with the exception of rage games-

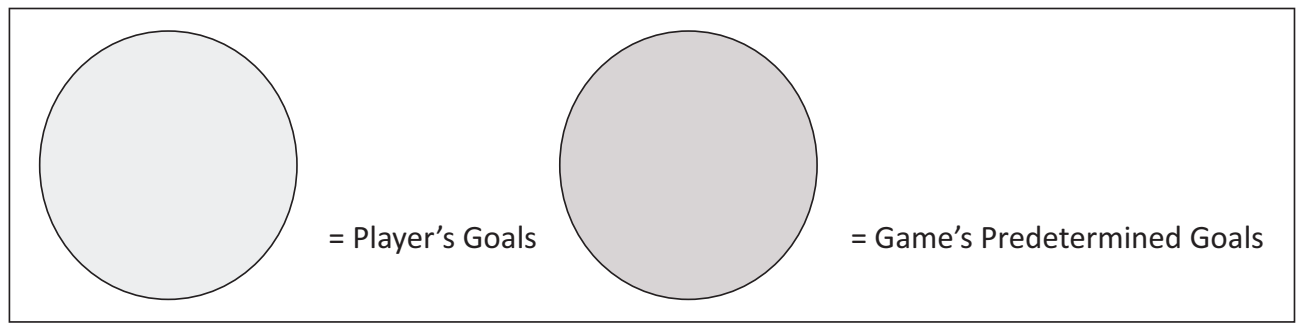

Situation A

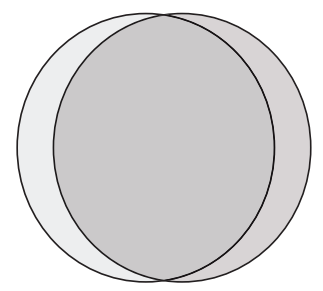

Situation B

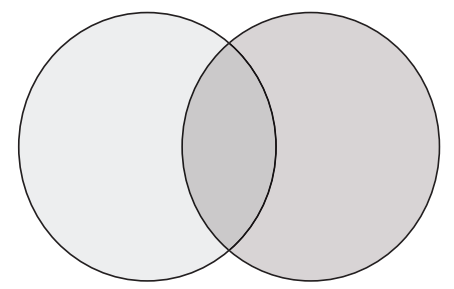

Situation C
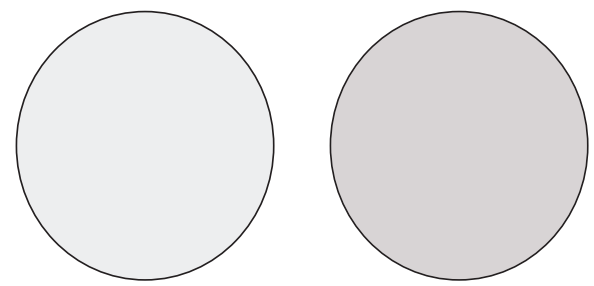

Figure 2. A visualization of players' goals and game developers' goals, and the various configurations of alignment or misalignment these can take. 
e.g., I Wanna Be the Guy and Trap Adventure 2-rare is the game players are expected to hate the whole way through (for a more complete discussion of these exceptions see Newman, 2018; Wilson \& Sicart, 2010), hence the at least partial alignment on the point of enjoyment. This would suggest that, if trolls go into the game experience expecting the game to provide some entertainment, they should start by attempting to meet or exceed the game's demands in order to experience the promised pleasure. The question then becomes: How do demands cause trolling when the player's and developer's goals are mostly aligned?

\section{Game Demands and Flow Theory Applied to Precursors Trolling Behaviour}

To answer the previous question, we have to turn to another important theory for game scholarship: flow theory (Nakamura \& Csikszentmihalyi, 2001). According to this theory, people can achieve a state of flow in which they feel focused, calm, and able to succeed when a person's skill at a task is perfectly balanced with the level of challenge that task presents (Chen, 2007; Stoll, 2019). It is essentially a dynamic state of optimal human functioning, and the theory has been applied to both traditional sports (Stoll, 2019) and video games (Chen, 2007). It is also the antithesis of the "tilt," described by gamers as a frequent precursor to negative trolling activity (Cook et al., 2018). Just as flow is a balancing act between challenge and skill, straying too far in one direction-either excessive skill or excessive challenge-can lead a person into the tilted headspace that often precedes trolling. If a level in a game is beyond the player's current skill level, for example, the player either has to develop their skills or try another level closer to their capabilities in order to achieve an optimal state. If the player does not make these changes, they risk becoming tilted once they pass their personal threshold of tolerance. It is important to note here that, just as one can be more or less tilted, this "optimal state" is ever-changing as the game enters into more or less challenging areas and as the player devel- ops their skills throughout the experience; there is no one optimal state, but rather what Chen (2006) calls a "fuzzy zone" in which players can experience this feeling of flow, allowing them to "work harder or work safer" to have fun. This concept of flow as a zone at the intersection of player skill and challenge is remarkably similar to that of cognitive and physical game demands. These, too, are an interaction between player and game (Bowman, 2018), and can produce the same kinds of behavioural outcomes (i.e., increased practice or changing levels in a game). Although arguably less researched than cognitive and physical demands, the same could be said of social and emotional demands in games, although instead of skill or proficiency interacting with challenge, it is more of a personal tolerance level and current state of being. People have differing preferences in terms of how they want to feel and how they want to interact with others (North, 1949), and games can push these boundaries in different directions. As a person's mood changes and interacts with a particularly poignant storyline segment in a game, this could theoretically push a person over the edge into unwanted tears. If the player is craving interaction with other people, a single-player run of a singleplayer game like Skyrim is unlikely to present enough social demand to give the player the kind of experience they desire. This could, however, change over time depending on the person's ever-shifting moods and desires at a given moment, as well as the game's different segments and challenges. Theoretically, we conceptualize flow and demand in much the same way.

Just as flow theory describes an optimal zone of functioning, I propose that demand theory is currently being used in much the same way: to describe the optimal range of demand in a game. The full extent of the demand dimension is presented in Figure 3. Demand levels within the middle range are what most game scholars interested in demand theory have studied. In this region, where demands are balanced with players' capacities, normal gameplay is expected. For cognitive and physical demands, which are arguably the most commonly researched in relation to media enjoyment (see

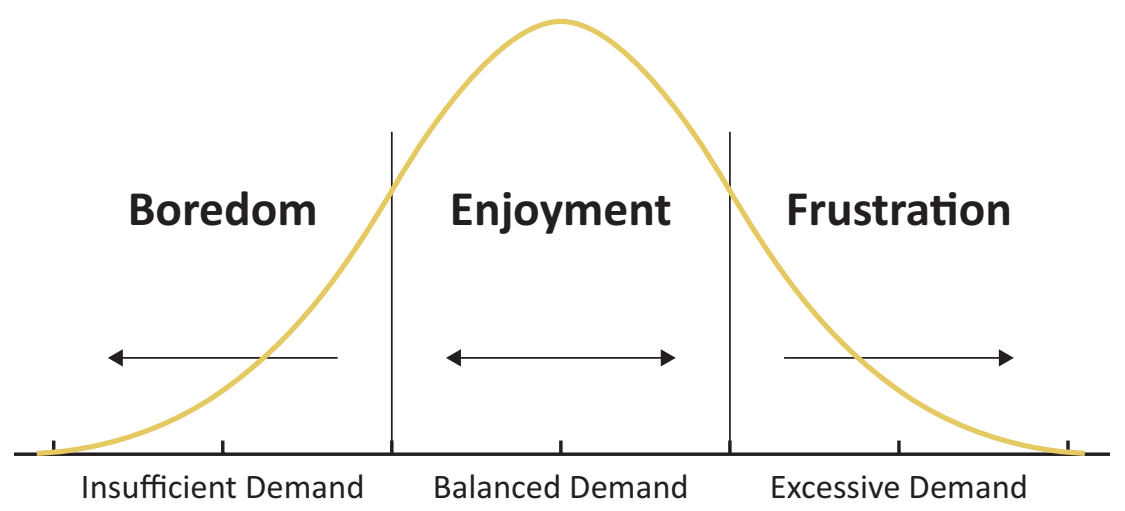

Figure 3. The full spectrum of demands as dimensions. Notes: The majority of gamers will fall into the middle section during the majority of their gameplay experience, which is what most demand research covers. The two extremes represent demand configurations that, if held for a significant period of time, could cause problematic behaviour (i.e., trolling). 
Sherry's, 2004, seminal article on the subject for more details), this is when the player is capable of using the game's mechanics effectively to attain theirs and the developers' goals, and this would produce normal gameplay. When it comes to social or emotional demands, I propose that this is when the level of demand produces the amount of interaction or emotive content (usually narrative) that allow the player to experience their desired emotions or desired amount of social interaction, while keeping their state of mind/being in a positive space. At the centermost range of the parabola would be the game experience of flow that game scholars often seek-Chen's (2006) "flow zone," in which players are able to meet the cognitive and physical challenges of the game and have a positive social and emotional experience playing the game. However, when these four demand types are either excessive or too limited when interacting with the player's skills and stateimbalanced-I hypothesize that players will be prone to exhibit trolling behaviour.

At either end of the curve presented in Figure 3 lie two of the primary motivations for trolling: boredom and frustration (Cook et al., 2018; Thacker \& Griffiths, 2012). At the left end of the curve, where the game demands are insufficient to meet the capacities of the player at the time, is boredom. Logically, if a game is too easy, it is unlikely to keep a player's attention (Patsis, Sahli, Verhelst, \& de Troyer, 2013). However, there is also evidence for this being a trolling trigger (Cook et al., 2018; Coyne et al., 2009; Thacker \& Griffiths, 2012). Cook et al. (2018) affirm that, in their sample, boredom was the second most popular catalyst to trolling behaviour. Trolls reported that, at a certain point, they became so proficient at the game that the game by itself was no longer exciting enough to keep their attention. From a flow perspective, the player capacities had likely increased to the point that the game was unable to create the challenge necessary for an optimal experience. Several other studies focusing on trolling also report that the number one global motivation for trolling is personal enjoyment, or as the trolls in Thacker and Griffiths' (2012) sample put it, "for the lulz." A particularly boring round of a game can, according to trolling literature, be the catalyst for a later round of trolling, highlighting the dynamic interaction between player and game over time.

On the other side of the parabola, however, is frustration. This is, of course, the opposite of boredom: when the game demands too much of the player, exceeding their capacities at the time, or pushing them too far emotionally or socially. Once again, we face the primary motivator for trolling behaviour: personal pleasure (Buckels et al., 2014; Cook et al., 2018; Shachaf \& Hara, 2010; Thacker \& Griffiths, 2012). Just as boredom can rob someone of a positive game experience, being too frustrated can cause the same outcome (see McGloin, Farrar, Krcmar, Park, \& Fishlock, 2016, for a full discussion of frustration and aggression in gameplay). This is also supported by evidence from trolling literature. In
Cook et al. (2018) taxonomy of trolling triggers, being "on tilt," gamer-speak for being in a negative headspace (Urban Dictionary, 2012), is listed alongside boredom as one of the top catalysts to trolling behaviour. It is worth noting that this frustration is often caused by losing a game, which is fundamentally a case of the game's cognitive and/or physical demands overwhelming the player's capacities. In other words, when the game's demands are excessive, the player can experience an emotional state that has been listed as a common precursor to trolling behaviour. That said, there is always room for individual differences, for just as flow is an interaction between skill and challenge, gameplay is an interaction between player and game, and no two players are exactly alike. Most games, even the most successful, have at least some moments of downtime (Pavlovich, 2014), while other games, such as the Dark Souls series, are beloved for their extreme degree of challenge (Rad, 2016). Regardless of players' differing tilting points, however, it would appear that excessive demands in a game can lead to frustration, which in turn can lead to trolling behaviour in-game.

\subsection{Demand Imbalance: Players vs. Games}

However, this all begs the question: Which particular game demands need to be excessive or lacking to cause trolling behaviour? Although this has yet to be formally researched, trolling literature does provide us with some clues. For example, one common thread running through most articles exploring trolling in games is the fact that trolls are often veteran players who prey on the inexperienced (Chesney et al., 2009; Cook et al., 2018; Thacker \& Griffiths, 2012). They claim to use their superior knowledge of the game to either misdirect newer players or to trick others into pointless arguments, wasting their time. This would suggest that trolls often have a high degree of mastery, which is to say that they have exceeded the cognitive and physical demands of the game and are adept users of the game's mechanics. Cook et al.'s (2018) trolls often claimed that the game itself no longer presented a challenge, and thus they applied their skills elsewhere in the game environment to assuage their desire for fun. This would suggest that the major demands that are out of whack are cognitive and physical-skillbased as opposed to state-based. However, trolls also talk about a generational gap in trolling, that trolls can be divided into two types: veterans and kids (Cook et al., 2018). The veterans say that they have exceeded the game's demands, while the kids, often called "squeakers" due to their high-pitched voices over voice chat (Rooster Teeth, 2011), appear to be consistently frustrated by the same demands. Their trolling is also said to differ, using more abrupt flaming and spamming, compared to veterans' more subtle tactics of misdirection (Cook et al., 2018). It remains unclear whether age or the result of differing demand imbalances (frustration vs. boredom) is the cause of these different trolling strategies, but the 
pattern does appear to both exist in extant literature and be explainable at least in part by cognitive and physical demand imbalance.

In the case of multiplayer games, the game itself often exerts a social demand as well. Although non-player characters can also exert social demands (Kryston, Novotny, Schmälzle, \& Tamborini, 2018; Peña, 2018), in competitive games like League of Legends or Counter Strike: Global Offensive which are known for their troll-filled communities (Cook et al., 2018; Cook, Conijn, Antheunis, \& Schaafsma, 2019), communication between players is an essential prerequisite to victory. As soon as teamwork becomes critical to winning, social interaction between players becomes a part of the game's demands. Incidentally, it is in these games that existing studies find the most heightened, and often aggressive, trolling behaviour (Blackburn \& Kwak, 2014; Cook et al., 2018; Kwak \& Blackburn, 2014; Kwak, Blackburn, $\&$ Han, 2015). This often consists of flaming (Cook et al., 2018, 2019; O'Sullivan \& Flanagin, 2003), although it can also take a behavioural form, such as feeding, which is purposely getting one's avatar killed in-game to advantage the other team. Although a formal causal relationship between the two has yet to be established, based on trolling accounts in literature examining multiplayer games, as the social demand of the game increases, the associated trolling appears to intensify accordingly (Cook et al., 2018; Kwak \& Blackburn, 2014). Given the fact that trolling takes place between human players in a virtual world (Cook et al., 2018), a situation in which trolling is caused by insufficient social demands is unlikely, as they are ever-present; however, there are games in which the social demands are variable. In massively-multiplayer online role playing games (MMORPGs), players can choose to play the game alone or with others in guilds, parties, or partnerships (Chen, Sun, \& Hsieh, 2008; Snodgrass et al., 2016), thus having the option to choose the amount of social interaction they desire instead of having a demand placed upon them. Reports of trolling behaviour in these games is also more varied in literature, from playfully pushing someone's avatar into water (Cook et al., 2018) to revealing real-life information about a player in-game (Chesney et al., 2009), also called "doxing" (MerriamWebster, n.d.). It remains to be tested whether this also varies as a function of whether the motivation is boredom or frustration, but again, the pattern itself does appear in trolling literature (Cook et al., 2018, 2019).

\subsection{Demand Imbalance: Players vs. Players}

All of that said, it is critical to understand that, in the case of trolling, the game itself is not the only source of demands. Though trolling work agrees on little, there is one element that all extant literature shares: Sociality (Buckels et al., 2014; Cook et al., 2018; Herring et al., 2002; Thacker \& Griffiths, 2012). Trolling cannot take place in a social vacuum; it requires a minimum of one troll and one victim, and due to its online nature, almost always includes several bystanders. As integral agents within the game environment, other players too exact demands on the player in question. These other players can be teammates, enemies, or be of no particular relation to the player in question, but they exist in the game world and have the same degree of agency as anyone else. Another player in a MMORPG, for example, could increase social demand by advertising an item to trade or a party looking for new members, while a skilled opponent in a multiplayer online battle arena (MOBA) could demand you sharpen your focus on the game, engaging more cognitive resources than a less-skilled opponent would require of you in order to achieve victory. On a bad day, a particularly vicious flame could be too emotionally demanding for a victim, who could theoretically respond with reciprocated aggression in chat or silent tears on the other side of the screen. In-game demands are always an interaction (Bowman, 2018), but interactions occur between players as well as players and the game itself. Thus, demand imbalance can come from two sources in a trolling situation - the game and the other players - and I hypothesize that both imbalances function according to the graph presented in Figure 3.

The mere presence of an "other" is enough to trigger a social demand (Peña, 2018), be it an artificial intelligence or otherwise, but social interaction between players in multiplayer games is usually quite intense (Smyth, 2007). Researchers have found that such interactions contain both socioemotional and task elements, meaning that players are concerned about how they and the other players are feeling, as well as completing the ingame goal (Peña \& Hancock, 2006). Existing research would thus suggest that players could exert social and/or emotional demands on their fellow players, usually by increasing the amount of the given demand. In the case of trolling, this would theoretically consist of the emotional demands of the troll pushing the victim's emotional state over the edge into negativity. However, there is also the possibility that other players can also exert physical or cognitive demands in a game as well. In a MOBA or firstperson shooter (FPS) game, for example, the other players on the opposing team essentially take the role of computerized enemies in a single-player game; the opponent's skill level becomes the demand, or in flow theory language, the challenge. By meeting or exceeding the physical and cognitive demands presented by the game, an opponent theoretically becomes the physical and cognitive demands players must meet or exceed themselves. The same could be hypothesized of player versus player modes in MMORPGs, although more often than not, studies show that gameplay in these types of games is cooperative (Martončik \& Lokša, 2015), which would mean that their skills would contribute to all players meeting the game's cognitive and physical demands collectively. In short, a player can exert social and emotional demands verbally via chat functions in a game, while they can either exert or relieve cognitive and physical demands through their gameplay. 
All that said, trolling itself is a form of social interaction, and thus also qualifies as a social demand to which players can respond. Trolling interactions have been shown in extant literature to use both excessive profanity and a high degree of game-specific jargon, suggesting that players are constantly being directed by or directing other players (Cook et al., 2019). It is also worth noting that these features are not exclusive to the troll; the same studies find that victims and bystanders seem to use as much profanity and jargon as trolls themselves (Cook et al., 2018, 2019). Existing research has also shown that trolling likely follows a cycle (Cook et al., 2018, 2019); victims are likely to become perpetrators, who in turn will likely create more victims, and so on and so forth. From the perspective of the demand framework, if players are excessively socially demanding, this would theoretically trigger trolling, which would in turn create further demand for other players, perpetuating the aforementioned cycle. When a person feels compelled to respond to perceived aggression, either verbally or behaviourally (Cook et al., 2018, 2019), theoretically, they have fewer resources to deal with the social, cognitive, and physical demands of the game, diminishing their capacities and thus likely tipping the demand balance in favour of frustration. Although a causal link has yet to be established, this pattern is reflected in trolling literature, as according to Cook et al. (2018) interviews with self-confessed trolls, being trolled first is the number one most popular catalyst to trolling behaviour. In short, victims appear to often feel compelled to respond to trolling behaviour, theoretically creating excessive social demand, which is likely to lead to frustration for victims, who may in turn go on to release that frustration via further trolling.

In fact, being a victim of trolling could not only be socially demanding, but also emotionally demanding, depending on the type of trolling and the victim's personality, personal vulnerabilities, and state of mind on the given day. For example, if a person takes the game at hand very seriously and personally, and a troll starts to purposely throw the game by feeding or broadcasting the team's positions to the enemy, this could feasibly take an emotional toll on the victim. That said, someone who was more relaxed about the experience, or who was in a particularly good mood, may not fall into the trolling cycle. Individual differences have been a major part of trolling research in recent years (Buckels et al., 2014; Craker \& March, 2016; March, Grieve, Marrington, \& Jonason, 2017; Sest \& March, 2017), and are an implicit part of demand theory in games as well, given demands' status as an interaction between unique players and unique games (Bowman, 2018). Personality scholars have yet to link individual differences to trolls in a gaming medium, but the labels they give to other online trollssadist and narcissist, to name a few (Buckels et al., 2014; Craker \& March, 2016)-suggest an emotional element. Sadism in particular paints an emotionally vampiric image of trolls, as if they feed off the emotional turmoil in their victims. The individual differences of trolling victims have also yet to be formally researched, but extant literature would suggest that they likely have lower capacities to deal with emotional demands, as trolls in most media forms have been shown to seek and obtain outrageous reactions from their victims (Buckels et al., 2014; Cook et al., 2018; Herring et al., 2002; Shachaf \& Hara, 2010). In any case, extant literature suggests that trolling does appear to have emotional impact in both victims and perpetrators, and has the potential to create emotional demand in a gaming setting.

\section{Conclusions and Future Directions}

In sum, although demand theory has typically been used to describe average and optimal gameplay, there is sufficient overlap with trolling literature to indicate that demand theory could also be used to explain deviant play. By extending demand theory to players as a source of demands and exploring the extremes of demands as dimensions, we can expand demand theory's applications beyond typical gameplay. When game demands fall out of the ideal range, the effects-boredom and frustrationhave been shown in extant literature to be causally linked to trolling behaviour (Buckels et al., 2014; Cook et al., 2018, 2019; Thacker \& Griffiths, 2012). In addition, when trolling is interpreted as an emotional or social demand, it also theoretically leads to further trolling, supporting the idea of the trolling cycle that is also present in trolling literature (Cook et al., 2018, 2019). For a field of research that is often derided for its atheoretical nature (see Cook et al., 2018), this application of demand theory is a promising step forward in marrying theory and empirical work in trolling literature.

Naturally, this is not the only framework that could 'solve' trolling, nor have demands been validated as a casual explanation for trolling as of yet. However, through the careful integration of existing theories like personality and flow with the demand framework, our understanding can continue to grow. Experiments can be designed to manipulate the level of various demands, for example, and see which manipulations cause which types of trolling. By manipulating the intensity of a flame in different gaming genres, for example, we could tease apart the emotional demands of a game from the emotional demands of other players and see which are more predictive of trolling outcomes in victims. Different personality characteristics can be evaluated in combination with different levels of different demands to determine which if any interactions are in play and how these produce different trolling types in trolls, and responses in victims and bystanders. There also remains the question of enjoyable vs. unenjoyable trolling, something that Paul, Bowman, and Banks (2015) have explored, but merits further testing in the light of this new connection with the demand framework. By testing these variables, we can better comprehend the exact precursors of trolling, and consequently learn how to prevent the 
kinds of trolling that insufficient or excessive demands could be creating.

There are also other perspectives to integrate, such as the ethnographic works of both Whitney Phillips (2015) and Kishonna Gray (2014), who explore how the unwritten rules inherent in Western society often dictate trolling practices in and beyond games. The cultural experience of trolling, whether it be in game you are playing or watching (Taylor, 2018), is a space that still needs exploring, as there are almost certainly variables in the humanities and beyond that can be added to strengthen our admittedly psychological explanations presented thus far. Even within the psychological perspective I have presented here, the concepts of frustration and boredom are still open for exploration, particularly in the context of emotional and social demands. Flow theory has been applied successfully in relation to cognitive and physical demands in the past (see Sherry, 2004), but its application to emotional and social demands in games is still new territory for games scholars and psychologists alike. In short, there is still more work to be done, but there is promise and there is a trajectory for researchers to explore. Armed with new variables to test, we are poised to begin to explain a problem that has plagued gamers and netizens for years.

\section{Acknowledgments}

The author thanks the editor and reviewers for their encouragement throughout the revision and publication process, as well as the Game Scholar Twitter community who served as an excellent resource for some tough questions along the way. She also sends out a heartfelt thank you to the rest of Twitter, who continue to serve as job security for trolling researchers everywhere.

\section{Conflict of Interests}

The author declares no conflict of interests.

\section{References}

Blackburn, J., \& Kwak, H. (2014). STFU NOOB! Predicting crowdsourced decisions on toxic behavior in online games. Paper presented at the 23rd International World Wide Web Conference, Seoul, South Korea.

Boot, W. R., Kramer, A. F., Simons, D. J., Fabiani, M., \& Gratton, G. (2008). The effects of video game playing on attention, memory, and executive control. Acta Psychologica, 129(3), 387-398. https://doi.org/ 10.1016/j.actpsy.2008.09.005

Bowman, N. D. (2018). The demanding nature of video game play. In N. D. Bowman (Ed.), Video games: $A$ medium that demands our attention (pp. 1-24). New York, NY: Taylor \& Francis.

Bowman, N. D., \& Tamborini, R. (2012). Task demand and mood repair: The intervention potential of computer games. New Media \& Society, 14(8), 1339-1357. https://doi.org/10.1177/1461444812450426

Bowman, N. D., Wasserman, J., \& Banks, J. (2018). Development of the video game demand scale. In $\mathrm{N}$. D. Bowman (Ed.), Video games: A medium that demands our attention (pp. 208-233). New York, NY: Taylor \& Francis.

Buckels, E. E., Trapnell, P. D., \& Paulhus, D. L. (2014). Trolls just want to have fun. Personality and Individual Differences, 67, 97-102. https://doi.org/10. 1016/j.paid.2014.01.016

Chen, C.-H., Sun, C.-T., \& Hsieh, J. (2008). Player guild dynamics and evolution in massively multiplayer online games. CyberPsychology \& Behavior, 11(3), 293-301. https://doi.org/10.1089/cpb.2007.0066

Chen, J. (2006). Flow in games: A Jenova Chen MFA thesis (Master's thesis). University of Southern California, Los Angeles, USA.

Chen, J. (2007). Flow in games (and everything else). Communications of the ACM, 50(4), 31-34. https:// doi.org/10.1145/1232743.1232769

Chesney, T., Coyne, I., Logan, B., \& Madden, N. (2009). Griefing in virtual worlds: Causes, casualties and coping strategies. Info Systems Journal, 19(6), 525-548. https://doi.org/10.1111/j.1365-2575.2009.00330.x

Cook, C. L., Conijn, R., Antheunis, M. L., \& Schaafsma, J. (2019). For whom the gamer trolls: A study of trolling interactions in the online gaming context. Journal of Computer-Mediated Communication, 2019. https:// doi.org/10.1093/jcmc/zmz014

Cook, C. L., Schaafsma, J., \& Antheunis, M. L. (2018). Under the bridge: An in-depth examination of online trolling in the gaming context. New Media \& Society, 20(9), 3323-3340. https://doi.org/10.1177/ 1461444817748578

Coyne, I., Chesney, T., Logan, B., \& Madden, N. (2009). Griefing in a virtual community: An exploratory survey of second life residents. Zeitschrift für Psychologie/Journal of Psychology, 217(4), 214-221. https:// doi.org/10.1027/0044-3409.217.4.214

Craker, N., \& March, E. (2016). The dark side of Facebook $^{\circledR}$ : The dark tetrad, negative social potency, and trolling behaviours. Personality and Individual Differences, 102, 79-84. https://doi.org/10.1016/j.paid. 2016.06.043

Daneels, R., Vandebosch, H., \& Walrave, M. (2019). Just for fun? Digital games' potential for meaningful media experiences among adolescents. Paper presented at the 69th Annual International Communication Association Conference, Washington, DC, USA.

Dynel, M. (2016). "Trolling is not stupid": Internet trolling as the art of deception serving entertainment. Intercultural Pragmatics, 13(3), 353-381. https://doi.org/ 10.1515/ipp.2016--0015

Eden, A., Ewoldsen, D. R., Lee, J., \& Beyea, D. (2018). Behavioral demands as behavioral affordances in video games. In N. D. Bowman (Ed.), Video games: A medium that demands our attention (pp. 92-107). New York, NY: Taylor \& Francis. 
Graham, E. (2019). Boundary maintenance and the origins of trolling. New Media \& Society, 21(9), 2029-2047. https://doi.org/10.1177/ 1461444819837561

Gray, K. L. (2014). Race, gender, and deviance in Xbox live: Theoretical perspectives from the virtual margins. New York, NY: Routledge.

Green, C. S. (2018). Video games and cognitive skills. In N. D. Bowman (Ed.), Video games: A medium that demands our attention (pp. 25-43). New York, NY: Taylor \& Francis.

Herring, S., Job-Sluder, K., Scheckler, R., \& Barab, S. (2002). Searching for safety online: Managing "trolling" in a feminist forum. The Information Society, 18(5), 371-384. https://doi.org/10.1080/ 01972240290108186

Johnson, M. R. (2019). Inclusion and exclusion in the digital economy: Disability and mental health as a live streamer on Twitch.tv. Information, Communication \& Society, 22(4), 506-520. https://doi.org/10.1080/ 1369118X.2018.1476575

Kryston, K., Novotny, E., Schmälzle, R., \& Tamborini, R. (2018). Social demand in video games and the synchronization theory of flow. In N. D. Bowman (Ed.), Video games: A medium that demands our attention (pp. 161-177). New York, NY: Taylor \& Francis.

Kwak, H., \& Blackburn, J. (2014). Linguistic analysis of toxic behavior in an online video game. Paper presented at the 1st "Exploration on Games and Gamers" workshop, Barcelona, Spain.

Kwak, H., Blackburn, J., \& Han, S. (2015). Exploring cyberbullying and other toxic behavior in team competition online games. Paper presented at "CHI: Crossings", Seoul, South Korea.

March, E., Grieve, R., Marrington, J., \& Jonason, P. K. (2017). Trolling on Tinder $^{\circledR}$ (and other dating apps): Examining the role of the dark tetrad and impulsivity. Personality and Individual Differences, 110, 139-143. https://doi.org/10.1016/j.paid.2017.01.025

Martončik, M., \& Lokša, J. (2015). Do World of Warcraft (MMORPG) players experience less loneliness and social anxiety in online world (virtual environment) than in real world (offline)? Computers in Human Behavior, 56, 127-134. https://doi.org/10.1016/j.chb. 2015.11.035

McGloin, R., Farrar, K. M., Krcmar, M., Park, S., \& Fishlock, J. (2016). Modeling outcomes of violent video game play: Applying mental models and model matching to explain the relationship between user differences, game characteristics, enjoyment, and aggressive intentions. Computers in Human Behavior, 62, 442-451. https://doi.org/10.1016/j.chb.2016.04. 018

Merriam-Webster. (n.d.). Dox. Merriam-Webster. Retrieved from https://www.merriam-webster.com/ dictionary/dox

Nakamura, J., \& Csikszentmihalyi, M. (2001). The concept of flow. In C. R. Snyder \& S. J. Lopez (Eds.), Handbook of positive psychology (pp. 89-105). Oxford: Oxford University Press.

Newman, J. (2018). Kaizo Mario Maker: ROM hacking, abusive game design and Nintendo's Super Mario Maker. Convergence, 24(4), 339-356. https://doi. org/10.1177/1354856516677540

North, R. D. (1949). An analysis of the personality dimensions of introversion-extroversion. Journal of Personality, 17, 352-368. https://doi.org/10.1111/j.14676494.1949.tb01219.x

O'Sullivan, P. B., \& Flanagin, A. J. (2003). Reconceptualizing 'flaming' and other problematic messages. New Media \& Society, 5(1), 67-93. https://doi.org/ $10.1177 / 1461444803005001908$

Patsis, G., Sahli, H., Verhelst, W., \& de Troyer, O. (2013). Evaluation of attention levels in a tetris game using a brain computer interface. In S. Carberry, S. Weibelzahl, A. Micarelli, \& G. Semeraro (Eds.), 21st International UMAP conference: User modeling, adaptation, and personalization, (pp. 127-138). Rome: Springer. Retrieved from https://link.springer.com/ content/pdf/10.1007\%2F978-3-642-38844-6.pdf

Paul, H., Bowman, N. D., \& Banks, J. (2015). The enjoyment of griefing in online games. Journal of Gaming and Virtual Worlds, 7(3), 243-258. https://doi.org/ 10.1386/jgvw.7.3.243_1

Pavlovich, M. (2014). Dimensions of games: Downtime. Games Precipice. Retrieved from http://www. gamesprecipice.com/downtime

Peña, J. (2018). A communication model of social demands in video games. In N. D. Bowman (Ed.), Video games: A medium that demands our attention (pp. 126-145). New York, NY: Taylor \& Francis.

Peña, J., \& Hancock, J. T. (2006). An analysis of socioemotional and task communication in online multiplayer video games. Communication Research, 33(1), 92-109. https://doi.org/10.1177/ 0093650205283103

Phillips, W. (2015). This is why we can't have nice things: Mapping the relationship between online trolling and mainstream culture. Cambridge, MA: MIT Press.

Possler, D., Klimmt, C., \& Raney, A. A. (2018). Gaming is awesome! A theoretical model on cognitive demands and the elicitation of aw during video game play. In N. D. Bowman (Ed.), Video games: A medium that demands our attention (pp. 74-91). New York, NY: Taylor \& Francis.

Rad, C. (2016, March 23). Dark Souls 3 is the hardest Dark Souls yet. IGN. Retrieved from https:// www.ign.com/articles/2016/03/23/dark-souls-3-isthe-hardest-dark-souls-yet

Rooster Teeth. (2011). Stop the squeakers in gaming!!! [Online discussion group]. Retrieved from https://roosterteeth.com/forum/general-gaming/ topic/2211106

Seering, J., Kraut, R., \& Dabbish, L. (2017). Shaping pro and anti-social behavior on Twitch through moderation and example-setting. In Proceedings of the 2017 
ACM conference on computer-supported cooperative work and social computing (pp. 111-125). New York, NY: ACM. Retrieved from http://delivery.acm.org/ $10.1145 / 3000000 / 2998277 /$ p111-seering.pdf?ip= 85.244.253.133\&id=2998277\&acc=CHORUS\&key= 4D4702B0С3Е38B35\%2E4D4702B0С3E38B35\% 2E4D4702B0C3E38B35\%2E6D218144511F3437 \&_acm__=1574261078_0723e8341e83379b17 af1f5e22f8f092

Sest, N., \& March, E. (2017). Constructing the cyber-troll: Psychopathy, sadism, and empathy. Personality and Individual Differences, 119, 69-72. https://doi.org/ 10.1016/j.paid.2017.06.038

Shachaf, P., \& Hara, N. (2010). Beyond vandalism: Wikipedia trolls. Journal of Information Science, 36(3), 357-370. https://doi.org/10.1177/ 0165551510365390

Sherry, J. L. (2004). Flow and media enjoyment. Communication Theory, 14(4), 328-347. https://doi.org/ 10.1111/j.1468-2885.2004.tb00318.x

Smyth, J. M. (2007). Beyond self-selection in video game play: An experimental examination of the consequences of massively multiplayer online roleplaying game play. CyberPsychology \& Behavior, 10(5), 717-721. https://doi.org/10.1089/cpb.2007.
9963

Snodgrass, J. G., Lacy, M. G., Dengah, H. F., Batchelder, G., Eisenhower, S., \& Thompson, R. S. (2016). Culture and the jitters: Guild affiliation and online gaming eustress/distress. Ethos, 44(1), 192. https://doi.org/ 10.1111/etho.12108

Stoll, O. (2019). Peak performance, the runner's high, and flow. In M. H. Ansel, S. J. Petruzello, \& E. E. Labbé (Eds.), APA handbook of sport and exercise psychology, volume 2: Exercise psychology (pp. 447-465). Washington, DC: American Psychological Association.

Taylor, T. L. (2018). Watch me play: Twitch and the rise of live game streaming. Princeton, NJ: Princeton University Press.

Thacker, S., \& Griffiths, M. D. (2012). An exploratory study of trolling in online video gaming. International Journal of Cyber Behavior, 2(4), 17-33. https://doi. org/10.4018/ijcbpl.2012100102

Urban Dictionary. (2012). Tilting. Urban Dictionary. Retrieved from https://www.urbandictionary.com/ define.php?term=Tilting

Wilson, D., \& Sicart, M. (2010). Now it's personal: On abusive game design. Paper presented at FuturePlay, Vancouver, Canada.

\section{About the Author}

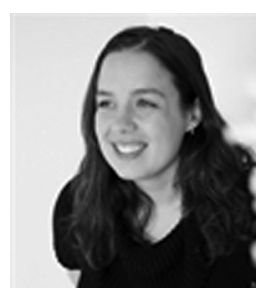

Christine L. Cook (MPhil, University of Cambridge) is a PhD Student in the Department of Communication and Cognition at Tilburg University. She researches online behaviour, with a specialization in so-called 'trolling' - a form of apparent deviance within cyberspace that takes place in a variety of contexts, including online games, forums, and social networking sites. 